\title{
LA PROTECCIÓN DE LOS DERECHOS FUNDAMENTALES Y LA MEJORA \\ DE LA CONVIVENCIA ESCOLAR A TRAVÉS DEL APRENDIZAJE TEÓRICO-PRÁCTICO DE LA FIGURA DEL DEFENSOR DEL PUEBLO
}

\section{THE PROTECTION OF FUNDAMENTAL RIGHTS AND THE IMPROVEMENT OF SCHOOL COEXISTENCE THROUGH THE THEORETICAL-PRACTICAL LEARNING OF THE FIGURE OF THE OMBUDSMAN}

\author{
Ma NiEVes Alonso García \\ Profa. Ayudante Doctora de Derecho Constitucional \\ marianieves.alonso@uil.es \\ Universidad Isabel I
}

\begin{abstract}
Resumen:
En la presente contribución se plantea una propuesta de innovación docente encaminada a dar a conocer a los alumnos la institución del Defensor del Pueblo a nivel estatal, y sus figuras homólogas en las Comunidades Autónomas, así como el Defensor del Pueblo Europeo en el ámbito de la Unión Europea como medio para manifestar determinadas problemáticas vinculadas con la convivencia escolar, entre otras. La presente propuesta se fundamenta en el paradigma socio-crítico referido a una metodología aprendizaje-servicio desde la que se plantea el diseño de una propuesta de innovación docente para promover en los estudiantes un acercamiento a la figura del Defensor del Pueblo. El trabajo se desarrollará con un grupo de cincuenta estudiantes de las asignaturas Derecho Constitucional y Derecho Administrativo del Grado en Derecho, con edades comprendidas entre 18 y 20 años, durante el curso académico 2019/2020. En esta línea, se pretende desarrollar una propuesta de intervención pedagógica que incorpore aspectos teórico-prácticos relativos a la figura del Defensor del Pueblo contemplando una serie de actividades centradas en los ámbitos de investigación, análisis, reflexión y resolución de casos prácticos, mientras se promueven a su vez acciones educativas transversales que proporcionarán a los estudiantes una visión más completa y positiva de sí mismos, de los demás y de su entorno. El objetivo de esta propuesta, al margen de acercar dicha institución a los estudiantes, se centra en la imperante necesidad de formar a ciudadanos conscientes de la importancia de velar por la protección de sus derechos fundamentales, además de hacerles partícipes del proceso transversal de respeto de los mismos en la esfera de sus relaciones sociales, las cuáles se desarrollan de forma esencial en el aula, espacio de construcción de redes y vínculos.
\end{abstract}

Palabras clave: innovación docente, Defensor del Pueblo, derechos fundamentales, enseñanza-aprendizaje.

\footnotetext{
Abstract:

REJIE Nueva época: Revista Jurídica de Investigación e Innovación Educativa

Núm.20, Junio 2019, pp. 49-59

[En línea] http://www.revistas.uma.es/index.php/rejie

Recibido: marzo 2019

Aceptado: mayo 2019
}

In the present contribution a teaching innovation proposal is proposed aimed at making known to the students the institution of the Ombudsman at the state level, and their counterpart figures in the Autonomous Communities, as well as the Ombudsman in the 
field of European Union as a means to manifest certain problems related to school coexistence, among others. The present proposal is based on the socio-critical paradigm referred to a learning-service methodology from which the design of a teaching innovation proposal is proposed to promote in the students an approach to the figure of the Ombudsman. The work will be developed with a group of fifty students of the subjects Constitutional Law and Administrative Law of the Degree in Law, with ages between 18 and 20 years, during the academic year 2019/2020. In this line, it is intended to develop a pedagogical intervention proposal that incorporates theoretical and practical aspects related to the figure of the Ombudsman contemplating a series of activities focused on the areas of research, analysis, reflection and resolution of practical cases, while promoting in turn transversal educational actions that will provide students with a more complete and positive view of themselves, others and their environment. The objective of this proposal, apart from bringing the institution closer to students, is centered on the prevailing need to train citizens aware of the importance of ensuring the protection of their fundamental rights, in addition to making them participants in the transversal process of respect for the same in the sphere of their social relations, which are developed in an essential way in the classroom, a space for the construction of networks and links.

Keywords: teaching innovation, Ombudsman, fundamental rights, teaching-learning.

Sumario: 1. Introducción: delimitación de objetivos. 2. Metodología y procedimiento de la propuesta. 2.1 Descripción metodológica. 2.2. Participantes. A. Docentes. Equipo multidisciplinar. B. Alumnos del primer curso del Grado en Derecho. 2.3 Diseño de la propuesta de innovación docente. A. Desarrollo de las sesiones teóricas. B. Desarrollo de las sesiones prácticas. 3. Resultados esperados. 4. Conclusiones. Bibliografía.

\section{Introducción: delimitación de objetivos.}

El artículo 27. 2 de la Constitución Española proclama que "la educación tendrá por objeto el pleno desarrollo de la personalidad humana en el respeto a los principios democráticos de convivencia y a los derechos y libertades fundamentales".

Esta reflexión planteada por nuestros constituyentes constituirá la esencia de la presente contribución en la cual se plantea una propuesta de innovación docente encaminada a dar a conocer a los alumnos la institución del Defensor del Pueblo a nivel estatal y sus figuras homólogas a nivel autonómico, así como la del Defensor del Pueblo Europeo en el ámbito de la Unión Europea.

Su objetivo principal, al margen de acercar dicha institución a los estudiantes, será formar a ciudadanos conscientes con la importancia de velar por la protección de sus derechos fundamentales, así como, por el respeto de los mismos en la esfera de sus relaciones sociales, fundamentalmente centradas en el aula como lugar en donde los alumnos construyen sus redes y vínculos.

El principal objetivo que se persiguen con esta propuesta de innovación se centra en contribuir a la mejora del proceso de enseñanza-aprendizaje sobre el fundamento de los artículos 27.2 y 54 de la Constitución Española favoreciendo los procesos educativos transversales. En los mencionados artículos se reconoce el derecho a la educación y su objeto, basado en el pleno desarrollo de la personalidad humana en el respeto a los principios democráticos de convivencia y a los derechos y libertades fundamentales y se 
configura la institución del Defensor del Pueblo como alto comisionado de las Cortes Generales, respectivamente ${ }^{1}$.

Dicho objetivo general se desarrollará a partir de objetivos específicos: realizar un reto instructivo en el ámbito de la educación que vincule a los docentes del ámbito universitario con las principales instituciones del Estado a partir de un proceso multidisciplinar de cooperación; orientar en el proceso de realización de quejas al Defensor del Pueblo; favorecer la autonomía del alumno de cara a manifestar un problema en la esfera de la convivencia escolar; fortalecer la coordinación entre estudiantes e instituciones públicas; desarrollar sesiones prácticas vinculadas con los contenidos teóricos difundidos a lo largo de las sesiones magistrales y consolidar un pensamiento reflexivo en los alumnos sobre la necesidad de proteger y respetar los derechos fundamentales a fin de consolidad una sociedad cívica, informada, crítica y seria.

Con todo ello se persigue el reto de aproximar la realidad social al aprendizaje, mejorando los recursos personales del alumno de manera que pueda contribuir a su desarrollo personal y en sus responsabilidades sociales.

\section{Metodología y procedimiento de la propuesta.}

\subsection{Descripción metodológica.}

La presente propuesta se fundamenta en el paradigma socio-crítico referido a una metodología aprendizaje-servicio ${ }^{2}$ desde la que se plantea el diseño de una propuesta de innovación docente para promover en los estudiantes un acercamiento a la figura del Defensor del Pueblo.

El trabajo se desarrollará con un grupo de cincuenta estudiantes con edades comprendidas entre 18 y 20 años, durante el curso académico 2019/2020. Para conocer las acciones, actitudes, opiniones y hechos específicos que fundamentan la manera de pensar y de actuar de los estudiantes se mantendrán varias conversaciones con los alumnos centradas en escalas de estimación respecto al ambiente escolar y, se realizará una prueba tipo test para evaluar el conocimiento que los alumnos poseen acerca de las figuras sobre las que versa la propuesta. Esta última prueba se realizará antes y después de la ejecución de la propuesta de Innovación.

En esta línea, se pretende desarrollar una propuesta de intervención pedagógica que incorpore aspectos teórico-prácticos relativos a la figura del Defensor del Pueblo contemplando una serie de actividades centradas en los ámbitos de investigación, análisis, reflexión y resolución de casos prácticos, mientras se promueven a su vez acciones educativas transversales que proporcionarán a los estudiantes una visión más completa y positiva de sí mismos, de los demás y de su entorno ${ }^{3}$.

\footnotetext{
${ }^{1}$ Para un amplio estudio doctrinal de la figura del Defensor del Pueblo, Vid. entre otros: PAU I VALL, F. (2016), El parlamento y el defensor del pueblo XXII Jornadas de la Asociación Española de Letrados de Parlamentos. Seminario Internacional: Modernización e Institucionalidad en el poder legislativo, Madrid: Tecnos.

2 MENDIA GALLARDO, R. (2012). "El Aprendizaje-Servicio como una estrategia inclusiva para superar las barreras al aprendizaje y a la participación", Revista Educación Inclusiva, 1:71-82.

3 ARANDA REDRUELLO, R.; CERRILLO MARTÍN, R.; DE LA HERRÁN GASCÓN, A.; DE MIGUEL BADESA. S.; GÓMEZ GARCÍA, M.; HERNÁNDEZ-CASTILLA, R.; IZUZQUIZA GASSET, D.; MURILLO TORRECILLA, F.J.; PÉREZ SERRANO, M.; RODRÍGUEZ-IZQUIERDO, R. $M^{a}$ y EGIDO GÁLVEZ, I. (Dir.) (2007), "El aprendizaje basado en problemas como innovación docente
} 
La propuesta de Innovación se desarrollará en diez sesiones enmarcadas en el horario lectivo correspondiente a la asignatura de "Derecho Constitucional" y "Derecho Administrativo". Dichas sesiones se impartirán durante el primer semestre del curso académico. Cada sesión tendrá carácter semanal y una duración de una hora, por lo que la propuesta constará de una duración total de diez semanas.

\subsection{Participantes.}

\section{A. Docentes: Equipo multidisciplinar.}

La presente propuesta de innovación estará constituida por un equipo multidisciplinar dentro del ámbito jurídico. El grupo de trabajo se formará por miembros pertenecientes a distintas áreas de conocimiento, íntimamente ligadas entre sí. De este modo, la propuesta engloba disciplinas directamente vinculadas con los principales objetivos de la misma. Por un lado, el Derecho Constitucional y por otro, el Derecho Administrativo. La primera de las ramas jurídicas tiene como objetivo velar por la existencia de una sociedad organizada sobre una base democrática y participativa, dentro de la cual adquiere especial relevancia la protección de los derechos fundamentales. La incorporación del Derecho Administrativo es significativa, ya que es la vertiente del Derecho público que se encarga de estudiar la organización y funciones de las instituciones del Estado. Desde este contexto, su contribución se centra en facilitar el conocimiento de la institución y en velar por la defensa de los derechos fundamentales que incida en la mejora de las relaciones personales entre los sujetos implicados.

La citada propuesta, se presentará enclavada, cómo se ha mencionado en líneas anteriores, en los contenidos curriculares de las asignaturas de Derecho Constitucional y Derecho Administrativo. La consolidación de conceptos en estas materias se revela complicada para los alumnos. Con el diseño y desarrollo de métodos alternativos más innovadores, basados en metodologías de aprendizaje-servicio ${ }^{4}$ como el aquí descrito se pretende que los estudiantes se muestren más participativos, reflexivos y proactivos, lo que conllevará con una alta probabilidad a que los mismos amplíen la complejidad de procesos lógicos desde sus propios intereses y necesidades ${ }^{5}$. Es en esta línea en la que se propone el desarrollo de la propuesta.

\section{B. Alumnos del primer curso del Grado en Derecho.}

La muestra con la que se pretende llevar a cabo la propuesta de innovación docente estará compuesta aproximadamente de cincuenta estudiantes de las asignaturas "Derecho Constitucional" y "Derecho Administrativo" integradas en el plan de estudios del primer curso del Grado en Derecho. Las edades de los participantes estarán comprendidas entre los 18 y 20 años.

en la universidad", Educación y futuro: revista de investigación aplicada y experiencias educativas, posibilidades y limitaciones, 16: 85-100.

${ }^{4}$ Se entiende por aprendizaje- servicio "una propuesta educativa que combina procesos de aprendizaje y de servicio a la comunidad en un único proyecto bien articulado en el que los participantes aprenden a la vez que trabajan en necesidades reales del entorno con la finalidad de mejorarlo" (PUIG, J.M.; MARTÍN, X. y BATLLE, R. (2007), Cómo iniciar un proyecto de aprendizaje y servicio solidario. Bilbao: Zerbikas Fundazioa).

5 TEJADA FERNÁNDEZ, J. (2013), "La formación de las competencias profesionales a través del aprendizaje servicio", Cultura y Educación: Culture and Education, 3: 285-294. 


\subsection{Diseño de la propuesta de innovación docente.}

El diseño de la propuesta se dividirá en las etapas descritas a continuación:

Etapa I. Diagnóstico del ambiente escolar y de las competencias en la materia propuesta. Esta etapa se desarrollará a través de conversaciones con los alumnos centradas en escalas de estimación respecto al ambiente escolar, así como de la realización de una prueba tipo test a través de la cual los docentes podrán evaluar el conocimiento que los alumnos poseen acerca de las figuras sobre las que versa la propuesta.

Etapa II. Sensibilización de la población escolar en el primer curso universitario. El objetivo consistirá en conseguir la implicación del alumno en la búsqueda e interpretación de información sobre las figuras de enseñanza propuestas.

Etapa III. Creación de la propuesta de Innovación, que se iniciará con la definición y exposición de los conceptos educativos básicos y finalizará con la ejecución del mismo como propuesta de solución a los problemas detectados. En esta etapa se llevarán a cabo las siguientes actividades:

- Construcción de una comunidad virtual de aprendizaje dónde el brainstorming y el intercambio de información útil se conviertan en técnicas de enseñanza entre los participantes. Esta comunidad virtual servirá también de punto de encuentro y comunicación siendo utilizada para la puesta en común de los avances relevantes;

- Estudio de opciones y planificación de actividades para el diseño del caso práctico,

- Búsqueda de información sobre el entorno y sus recursos para la construcción del caso práctico

- Diseño del caso práctico. Diagnóstico del problema.

- Búsqueda de soluciones y elaboración de la propuesta final.

- Presentación pública del caso práctico ante sus compañeros y los docentes.

Etapa IV. Ejecución de la propuesta de Innovación partiendo del diseño y asignación de actividades para aplicar; el seguimiento de la implementación de las mismas, discusión de avances y resultados en el aula, y presentación pública.

Etapa V. Evaluación y seguimiento de la propuesta constatando el aprendizaje de los estudiantes mediante la aplicación del instrumento de medida inicial, el análisis en el aula de las propuestas solventes a problemáticas del entorno; la sistematización de experiencias relativas al proyecto, la presentación de la experiencia de aprendizajeservicio en el aula y el análisis de los resultados del proyecto a partir de herramientas del desarrollo del pensamiento.

\section{A. Desarrollo de las sesiones teóricas.}

En las sesiones teóricas se enseñará a los alumnos la regulación y funciones de la institución del Defensor del Pueblo. Entre otros contenidos, se enseñará a los alumnos acerca de esta figura, que se encuentra constitucionalmente reconocida en el artículo 54 y fue creada por la Ley Orgánica 3/1981, de 6 de abril, del Defensor del Pueblo. El 
artículo 12 de la citada legitima la existencia de órganos similares en las Comunidades Autónomas ${ }^{6}$.

El objetivo de estas sesiones teóricas consiste en que el alumno aprenda que la principal función del Defensor del Pueblo es velar porque se garantice a los ciudadanos el derecho a una buena administración y cuidar especialmente de proteger y defender los derechos de los menores y de todas las personas en peligro de exclusión social.

El Defensor del Pueblo es un Alto Comisionado de las Cortes Generales y, en consecuencia, debe elevar a éstas un informe anual. A su vez, entre sus funciones de salvaguardia de la actividad de la Administración, se encuentran las propias recomendaciones que le puede efectuar. Estos informes y recomendaciones no han sido sólo instrumentos básicos sobre los que se ha cimentado el prestigio de esta institución, han constituido ocasionalmente impulsos de mejora legislativa o de actuación de la Administración.

En cuanto a la defensa de los derechos comprendidos en el Título I de nuestra vigente Carta Magna, tal y como establece el artículo 54 CE y el artículo 1 de su Ley Orgánica, se articula por medio del control de la Administración. La mayor parte de las actuaciones del Defensor del Pueblo se orientan a asegurar esta fiscalización de la Administración en aras a cumplir los principios que de ella se predican: eficacia, jerarquía, coordinación y sometimiento pleno a la ley y al derecho ${ }^{7}$.

Asimismo, será relevante que los alumnos conozcan la existencia de figuras homólogas a nivel autonómico, consecuencia de la descentralización política interna de administraciones autonómicas que ha originado la creación de defensores del pueblo específicos para cada una de las Comunidades Autónomas; así como la existencia del Defensor del Pueblo Europeo, fruto del proceso de integración europea.

En cuanto a las funciones y naturaleza del Defensor del Pueblo Europeo, se transmitirá a los alumnos que esta institución aparece en el Derecho de la Unión Europea como una persona física, elegida y nombrada por el Parlamento Europeo, para que reciba e instruya las reclamaciones de los ciudadanos europeos relativas a casos de mala administración en la actuación de las instituciones, organismos y órganos de la Unión, e informe de sus investigaciones y resultados tanto al ciudadano, como al órgano concernido y al Parlamento Europeo.

Su fundamentación jurídica se sustenta en los artículos 20, 24 y 228 del Tratado de Funcionamiento de la Unión Europea y artículo 43 de la Carta de los Derechos Fundamentales de la Unión Europea. El estatuto y las funciones del Defensor del Pueblo se fijaron mediante una Decisión del Parlamento Europeo de 9 de marzo de 1994 (DO L 113 de 4.5.1994, p. 15), modificada por las Decisiones del Parlamento de 14 de marzo de 2002 (DO L 92 de 9.4.2002, p. 13) y de 18 de junio de 2008 (DO L 189 de 17.7.2008, p. 25) y adoptada previo dictamen de la Comisión y con la aprobación del Consejo. Esta Decisión fue objeto de disposiciones de aplicación adoptadas por el propio Defensor del Pueblo Europeo. Las modalidades de su elección y destitución se establecen, por su parte, en los artículos 219 a 221 del Reglamento interno del Parlamento Europeo.

\footnotetext{
${ }^{6}$ ÁLVAREZ VÉLEZ, Ma I. (2018), "El Defensor del Pueblo y los defensores autonómicos: instituciones de garantía de los derechos" en ÁLVAREZ VÉLEZ, M ${ }^{\mathrm{a}}$ I., VIDAL PRADO, C. (Coords.): $L a$ Constitución Española: 1978-2018, Madrid: Francis Lefebvre.

7 ALGUACIL GONZÁLEZ-AURIOLES, J., REVIRIEGO PICÓN, F. (2010), "Repertorio bibliográfico sobre el Defensor del Pueblo", Teoría y Realidad Constitucional, 26: 563-614.
} 
Se trata de una autoridad de disuasión que, a través de sus tres medios esenciales de actuación, como son la investigación, la recomendación y la publicidad de sus críticas, trata de influir y de persuadir, ayudar y orientar, y no de imponer, vencer u obligar. No es un juez, ni un tribunal, ni puede dar órdenes, ni modificar o anular actos administrativos. Ahora bien, el carácter no vinculante de sus decisiones no obsta para que obtenga el respeto de los ciudadanos si finalmente resulta eficaz en sus actuaciones, especialmente, en las que supongan la denuncia de actos que lesionen derechos de los ciudadanos o de búsqueda de soluciones a problemas particulares.

Está legitimado para presentar una reclamación al Defensor del Pueblo Europeo todo ciudadano de la UE o toda persona física o jurídica -empresa, asociación, fundación...que resida o tenga su sede social en un Estado miembro de la Unión. Lo más efectivo es presentar la reclamación través de la página web del Defensor del Pueblo ${ }^{8}$. Este derecho de reclamación se ha consolidado en la práctica institucional como una vía alternativa a la del recurso ante el juez comunitario para la defensa de los intereses por parte de los ciudadanos; una vía alternativa, es decir, extrajudicial, que responde a criterios específicos y no persigue los mismos que la vía judicial. El procedimiento de presentación es muy flexible y, además, gratuito. La reclamación puede formularse por escrito, en cualquiera de las lenguas reconocidas por el Tratado de la Unión Europea, utilizando un formulario que facilita la Secretaría del Defensor del Pueblo, pudiendo hacerse llegar por correo (postal o electrónico) o por fax. El Estatuto del Defensor del Pueblo exige que se presente en un plazo de dos años desde que se tuvo conocimiento de los hechos, pero la aplicación estricta de este requisito es discutible e incluso inoperante. La reclamación debe incluir con claridad el acto objeto de la misma, y la identidad del reclamante, aunque el Defensor del Pueblo podrá clasificarla como confidencial si lo considera necesario.

Uno de los aspectos más importantes de la misión del Defensor del Pueblo en todos sus niveles consiste en generar confianza a través del diálogo con los ciudadanos. Parte de la función de un Defensor del Pueblo en cualquier democracia moderna es ocuparse de que la administración pública se oriente al servicio a los ciudadanos e intente satisfacer sus cada vez más elevadas expectativas 9 .

En definitiva, los alumnos deben ser partícipes del entorno que les rodea estando al corriente de que cuando un ciudadano presente cierto descontento con una institución o se atente a sus derechos constitucionalmente garantizados, se le debe brindar la oportunidad de corregir tal situación. Y es el Defensor del Pueblo la institución donde se debe inferir la reclamación para que tenga conocimiento del problema.

\section{B. Desarrollo de las sesiones prácticas.}

Las sesiones prácticas se desarrollarán en las últimas sesiones de la propuesta de innovación. Para ello, el docente expondrá a sus alumnos un marco contextual común al hilo del mismo y a partir del cual se irán conformando las distintas actividades prácticas en función de los avances perpetrados en el aula.

\footnotetext{
${ }^{8} \mathrm{http}: / /$ www.ombudsman.europa.eu.

${ }^{9}$ Sobre este particular, Vid. entre otros, SALVADOR MARTínEZ, M. (2015), "El Defensor del Pueblo europeo" en SÁNCHEZ GUTIÉRREZ, M. / SALVADOR MARTÍNEZ, M. (Coords.): Lecciones básicas de derecho e instituciones de la Unión Europea, Madrid: Manuales UEX ON_LINE.
} 
Es fundamental que el docente centre al alumno en el estado actual de la cuestión destacando que, en los últimos años, han aumentado los conflictos de convivencia escolar, tanto vinculados a agresiones como vinculados a los problemas de integración de los alumnos de origen extranjero.

El desarrollo de esta parte se distribuirá en las cinco sesiones prácticas que se describen a continuación:

Sesión práctica 1: "Pongamos en práctica todo aquello que hemos aprendido". En esta sesión, el docente expondrá 10 casos a los alumnos y dejará que ellos discutan acerca de la posibilidad de presentárselos al Defensor del Pueblo. De este modo y a partir de la discusión espontánea, los alumnos tendrán claro qué quejas (traducidas a la práctica) pueden elevarse al Defensor del Pueblo.

Sesión práctica 2: "Elijamos un Defensor del Pueblo". El docente dividirá la clase en seis grupos. De entre los miembros de cada grupo se elegirá, por consenso, a un alumno que actuará como Defensor del Pueblo resolviendo con ayuda de sus compañeros de grupo la queja planteada por otro de los grupos en los que se ha dividido el aula. El docente no intervendrá hasta el final de la sesión cuando potencie los éxitos de los grupos y fortalezca las debilidades mostradas en el desarrollo de la sesión.

Sesión práctica 3: Lluvia de ideas e inicio de redacción de la queja común del aula para elevar al Defensor del Pueblo.

Sesión práctica 4: Perfil de la queja y preparación de la exposición. Para ello, los alumnos deberán valerse de los recursos facilitados en la web del Defensor del Pueblo. (https://www.defensordelpueblo.es/)

Sesión práctica 5: El alumno que haya sido elegido como Defensor de Pueblo junto con los compañeros de su grupo, deberá considerar, conforme a la queja, qué tipo de resolución deberá formular a las administraciones (una recomendación en la que se propone que se modifique la interpretación de una norma o incluso la creación de una nueva y tiene un alcance general, una sugerencia de modificación de una actuación concreta, que afecta a únicamente a un ciudadano particular o a una comunidad concreta; un recordatorio de la obligación de cumplir una exigencia legal o una advertencia en la que comunica la existencia de una situación de hecho o práctica que precisa de mejora o, en último lugar, una solicitud de recurso de inconstitucionalidad ante el Tribunal Constitucional) y los argumentos para su concreta resolución, que serán expuestos en el aula.

El desarrollo de esta experiencia práctica podrá extrapolarse a los problemas de convivencia escolar generados en las aulas en todos los niveles educativos, en donde se fomentará la resolución consensuada de los conflictos.

La adquisición de conocimientos debe devenir en la formación de una sociedad cívica, informada, crítica y seria que se sustente en el pleno respeto de los derechos fundamentales.

\section{Resultados esperados.}

Con la propuesta de innovación descrita a lo largo de estas líneas se pretende mejorar la calidad del proceso educativo usando el material interactivo generado, así como la consolidación de un grupo de investigación mixto constituido por disciplinas afines, que en su actuación conjunta fomenten el mejor aprendizaje por parte del alumno. 
Asimismo, el trabajo colaborativo de los alumnos supondrá la administración de grupos de clase a través de la propuesta educativa y la construcción de entornos personales de aprendizaje.

Fruto de esta experiencia, se potenciará el trabajo cooperativo y se impulsará la ruptura del distanciamiento entre los ciudadanos y las instituciones del Estado.

La actividad permitirá al alumno desarrollar las competencias generales de las asignaturas Derecho Constitucional y Derecho Administrativo a través del conocimiento de una parte de los contenidos curriculares de las materias mencionadas ${ }^{\mathbf{1 0}}$.

Los alumnos se implicarán en la búsqueda, obtención, procesamiento y comunicación de información (oral, impresa, audiovisual, digital o multimedia) para transformarla en conocimiento y aplicarla en los procesos de enseñanza y aprendizaje en las materias

En esta misma línea, se adquirirán estrategias para estimular el esfuerzo del estudiante y promover su capacidad para aprender por sí mismo y con otros, y desarrollar habilidades de pensamiento y de decisión que faciliten la autonomía, la confianza e iniciativa personales.

Como reto final de esta propuesta se pretenderá fomentar el espíritu crítico, reflexivo y emprendedor del alumno y el desarrollo de habilidades de aprendizaje que les permitan continuar estudiando de un modo que habrá de ser en gran medida autodirigido y autónomo.

Para conocer si a través de la propuesta los alumnos han adquirido los conocimientos esperados sobre la figura del Defensor del Pueblo, se realizará una prueba tipo test después de la ejecución de la misma para evaluar, si respecto a la prueba realizada con carácter previo a la puesta en práctica de la propuesta, ha contribuido a mejorar el conocimiento que los alumnos tienen sobre esta figura y sus funciones.

\section{Conclusiones.}

La Declaración final de la Conferencia Mundial de Educación Superior organizada por la Unesco en 1998 estableció que "La educación superior ha de reforzar sus funciones de servicio a la sociedad y, más concretamente, sus actividades orientadas a erradicar la pobreza, la intolerancia, la violencia, el analfabetismo, el hambre, el deterioro del medio ambiente y las enfermedades, sobre todo con un planteamiento interdisciplinario y transdisciplinario para analizar los problemas y las dudas planteados".

Tomando como punto de partida esta reflexión, el objetivo perseguido en esta propuesta es fomentar una educación en valores para desarrollarse en un entorno de respeto a los derechos fundamentales a través del aprendizaje de las instituciones del Estado. La enseñanza debe perseguir a través de la adquisición de conocimientos la formación de una sociedad cívica, informada, crítica y seria.

El aula representa la esfera en la que los alumnos desarrollan sus relaciones sociales, constituyendo un espacio de construcción de redes y vínculos. Los trabajos colaborativos conducen a una dinámica en la que se engloban ideales, intereses, motivaciones y necesidades que rigen la conducta y las decisiones.

\footnotetext{
${ }^{10}$ Vid. sobre este particular, CANO GARCÍA, E. (2015), Evaluación por competencias en educación superior, Madrid: La Muralla.
} 
Es fundamental que los alumnos aprendan a analizar, programar, evaluar y trabajar en equipo con el resto del grupo, negociar y distribuir tareas, adquirir compromisos y tomar decisiones.

A través de la experiencia teórica de esta propuesta, el alumno se acercará a una institución fundamental de nuestro ordenamiento jurídico, profundizando en su conocimiento a su posterior puesta en práctica. El desarrollo práctico fomentará la convivencia pacífica en el aula y la resolución de conflictos a través de la mediación y las buenas prácticas ${ }^{11}$.

\section{Bibliografía.}

-ALGUACIL GONZÁLEZ-AURIOLES， J.; REVIRIEGO PICÓN， F. (2010), "Repertorio bibliográfico sobre el Defensor del Pueblo", Teoría y Realidad Constitucional, 26: 563-614.

-ÁLVAREZ VÉLEZ, M ${ }^{\mathrm{a}}$ I. (2018), "El Defensor del Pueblo y los defensores autonómicos: instituciones de garantía de los derechos" en ÁLVAREZ VÉLEZ, M ${ }^{\mathrm{a}}$ I., VIDAL PRADO, C. (Coords.): La Constitución Española: 1978-2018, Madrid: Francis Lefebvre.

-ARANDA REDRUELLO, R.; CERRILLO MARTÍN, R.; DE LA HERRÁN GASCÓN, A.; DE MIGUEL BADESA. S.; GÓMEZ GARCÍA, M.; HERNÁNDEZCASTILLA, R.; IZUZQUIZA GASSET, D.; MURILLO TORRECILLA, F.J.; PÉREZ SERRANO, M.; RODRÍGUEZ-IZQUIERDO, R. Ma y EGIDO GÁLVEZ, I. (Dir.) (2007), "El aprendizaje basado en problemas como innovación docente en la universidad", Educación y futuro: revista de investigación aplicada y experiencias educativas, posibilidades y limitaciones, 16: 85-100.

-CANO GARCÍA, E. (2015), Evaluación por competencias en educación superior, Madrid: La Muralla.

-GONZÁLEZ MORENO, B. (2017), "La tutela de los derechos y libertades por los defensores del pueblo: del límite que representa la función jurisdiccional a la ampliación de las funciones mediadoras de los defensores" en MÍGUEZ MACHO, L. y GONZÁLEZ MORENO, B. (Coords.), Resolución judicial y extrajurisdiccional de conflictos en el proceso de modernización de la administración de justicia, Madrid: Tecnos.

-MENDIA GALLARDO, R (2012), "El Aprendizaje-Servicio como una estrategia inclusiva para superar las barreras al aprendizaje y a la participación", Revista Educación Inclusiva, 1:71-82.

\footnotetext{
${ }^{11}$ Sobre esta función del Defensor del Pueblo, Vid. entre otros: GONZÁLEZ MORENO, B. (2017), "La tutela de los derechos y libertades por los defensores del pueblo: del límite que representa la función jurisdiccional a la ampliación de las funciones mediadoras de los defensores" en MÍGUEZ MACHO, L. y GONZÁLEZ MORENO, B. (Coords.), Resolución judicial y extrajurisdiccional de conflictos en el proceso de modernización de la administración de justicia, Madrid: Tecnos.
} 
-PAU I VALL, F (2016), El parlamento y el defensor del pueblo. XXII Jornadas de la Asociación Española de Letrados de Parlamentos. Seminario Internacional: Modernización e Institucionalidad en el poder legislativo, Madrid: Tecnos.

-PÉREZ-UGENA COROMINA, M. (2017), “Garantía del derecho a la tutela judicial efectiva en los sistemas principales de resolución de conflictos alternativos: arbitraje y mediación". Estudios de Deusto: revista de la Universidad de Deusto, 62, 1: 159-189.

-PUIG, J.M., MARTÍN, X. Y BATLLE, R. (2007), Cómo iniciar un proyecto de aprendizaje y servicio solidario. Bilbao: Zerbikas Fundazioa.

-SALVADOR MARTÍNEZ, M. (2015), "El Defensor del Pueblo europeo" en SÁNCHEZ GUTIÉRREZ, M. I SALVADOR MARTÍNEZ, M. (Coords.): Lecciones básicas de derecho e instituciones de la Unión Europea, Madrid: Manuales UEX ON_LINE.

-TEJADA FERNÁNDEZ, J. (2013), "La formación de las competencias profesionales a través del aprendizaje servicio", Cultura y Educación: Culture and Education, 3: 285294. 